\title{
Vollremission eines zerebral, pulmonal und hepatogen metastasierten malignen Melanoms unter Fotemustin mit passagerem Auftreten eines demenziellen Syndroms unter Valproinsäure
}

\author{
Long-Term Complete Remission of Cerebral, Pulmonary and Hepatic Metastases by Malignant Melanoma \\ under Fotemustin with Reversible Dementia Syndrome Due to Valproic Acid
}

Autoren

Institut

\section{Eleftheriadis, R. Stadler}

Dermatologie am Johannes Wesling Klinikum Minden
Bibliografie

DOI http://dx.doi.org/

10.1055/s-0041-107175

Online-Publikation: 11.12 .2015

Akt Dermatol 2016; 42: 31-34

(c) Georg Thieme Verlag KG

Stuttgart · New York

ISSN 0340-2541

\section{Korrespondenzadresse}

Vlasios Eleftheriadis

Hautklinik Johannes Wesling

Klinikum Minden

Hans-Nolte-Straße 1

32429 Minden

Vlasios.Eleftheriadis@

muehlenkreiskliniken.de

\section{Zusammenfassung \\ $\nabla$}

Eine 63-jährige Frau erleidet in 4/2010 erstmalig einen generalisierten epileptischen Anfall durch eine singuläre Hirnmetastase bei vor 40 Jahren exzidiertem malignem Melanom in der linken Ellenbeuge.

Neben der zerebralen Filia finden sich im Staging auch Metastasen in Lunge und Leber. Die Hirnfilia und Lungenmetastasen werden reseziert, zudem erfolgt die medikamentöse Versorgung mit Valproat und in 8/2010 die Einleitung einer Chemotherapie mit Fotemustin.

Nach der 10. Gabe Fotemustin kommt es zu einem plötzlich einsetzenden und rasch progredienten körperlichen und kognitiven Verfall mit demenziellen Symptomen sowie körperlicher Schwäche und Tremor. In 10/2011, nach 13 Gaben Fotemustin, erfolgt ein stationärer Aufenthalt in der Neurologischen Klinik. Nach eingehender Diagnostik wird ein Progress der Grunderkrankung als Ursa-

\section{Anamnese}

\section{$\nabla$}

Eine 63-jährige Patientin wurde nach einem Krampfanfall am 5.4.2010 von ihrem Ehemann im Schlafzimmer mit Blickwendung nach links gefunden. Am 6.4.2010 erfolgte die Aufnahme in der Neurologischen Klinik des Johannes Wesling Klinikums. cCT und MRT-Hirnschädel zeigten eine Raumforderung hoch frontoparietal mit perifokalem Ödem rechts ( $\bullet$ Abb. 1). Bei der Patientin wurde ca. 1970 ein malignes Melanom von der linken Ellenbeuge exzidiert. Genauere histologische Angaben lagen nicht vor (Melanomtyp, Gesamttumordicke). che für den Verfall ausgeschlossen. Es findet sich auch sonst kein Hinweis für die Ursache der Demenz. Verweisend auf Parallelen zu Fallberichten wird die Diagnose einer Fotemustin-induzierten Neurotoxizität formuliert.

Die bereits pausierte Chemotherapie mit Fotemustin wird nicht fortgeführt. Bei Fortschreiten des Verfalls stellt sich die Patientin auswärts in einem Epilepsiezentrum vor. Hier wird die antikonvulsive Therapie von Valproat auf Levetiracetam umgesetzt. Nach der Umstellung kommt es zu einem kompletten Rückgang der demenziellen Symptomatik.

Die beschwerdefreie Patientin stellt sich seither alle drei Monate zum Staging vor. $1 \frac{1}{2}$ Jahre nach Beendigung der Fotemustintherapie befindet sich die Patientin in Vollremission. Der vorgestellte Fall ist ein Beispiel für eine erfolgreiche Therapie mit Fotemustin. Die zunächst befürchtete Nebenwirkung der Neurotoxizität konnte in diesem Fall einem anderen Medikament zugeordnet werden.

\section{Diagnoseblock}

Symptomatische fokale Epilepsie mit tonisch-klonischen Anfällen bei Z.n. Resektion einer Metastase rechtsfrontal, arterielle Hypertonie.

\section{Therapie und Verlauf \\ $\nabla$}

Am 12.4.2010 erfolgte eine MR-navigierte Tumorentfernung über eine osteoplastische Kraniotomie rechts frontal. Aus der Histologie bestätigte sich der Verdacht einer Melanommetastase.

Die postoperative cCT-Kontrolle zeigte einen regelrechten postoperativen Befund ohne Hinweis auf einen Resttumor ( $\mathbf{A b b}$. 2).

Neurologischerseits erfolgte die Einleitung einer antiepileptischen Therapie mit Valproat. In der PET-CT-Untersuchung imponierten 3 metastasenverdächtige Rundherde im rechten Unter- 


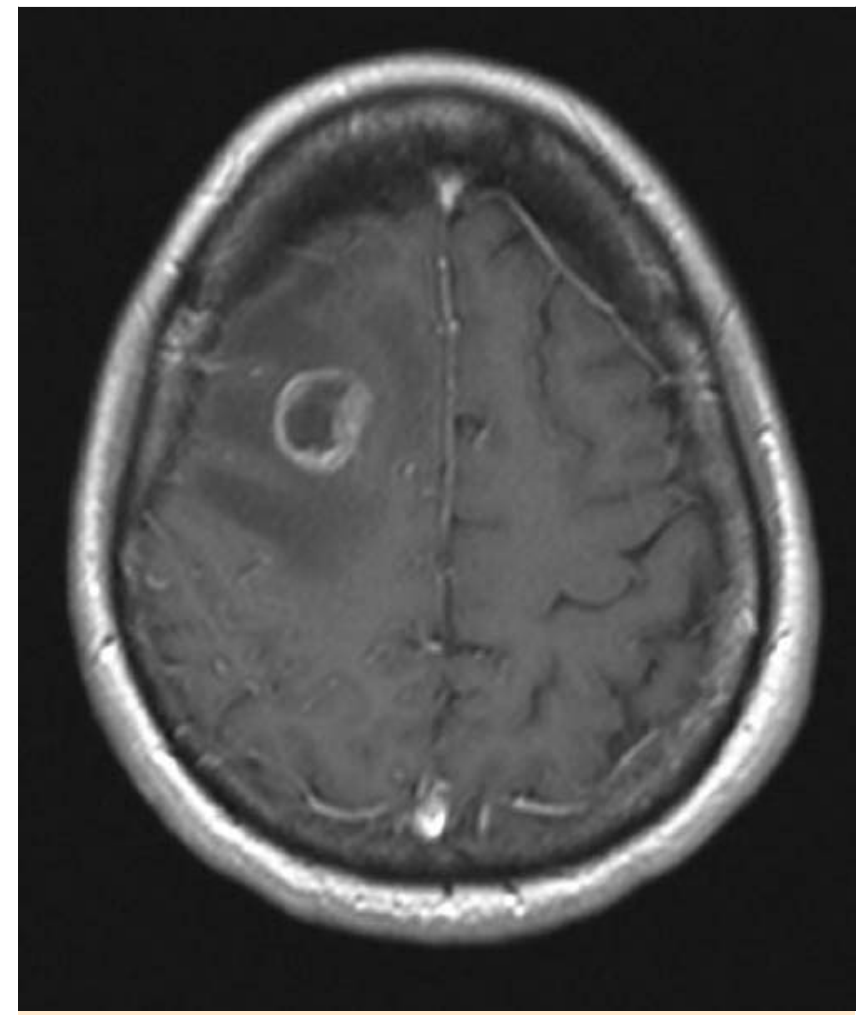

Abb. 1 Schädel-MRT vom 8.4.2010: T1-Wichtung mit KM-Gabe.

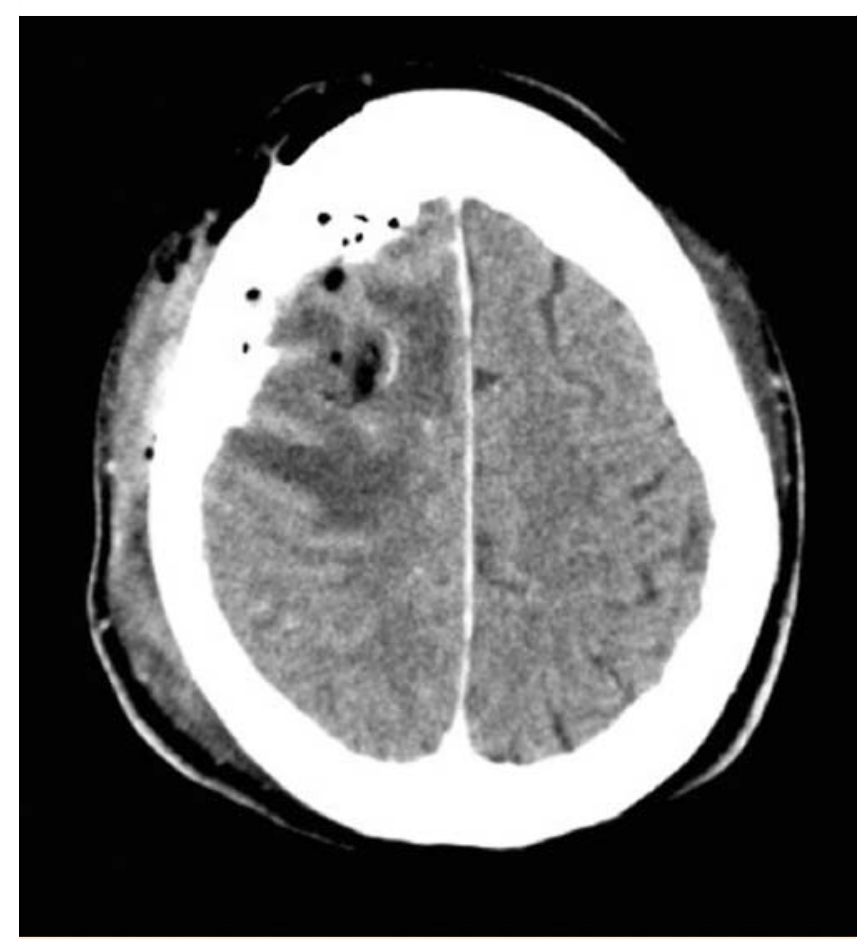

Abb.2 Schädel-CT vom 13.4.2010 nach Metastasenresektion.

und Oberlappen. Nach der TNM-Klassifikation wurde die Patientin im Stadium pTx N0 M1c, klinisch Stadium IV, eingestuft. Der

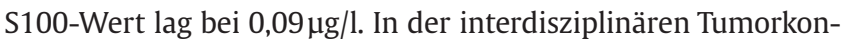
ferenz wurde die operative Versorgung der Lungenmetastasen vorgeschlagen ( $\bullet$ Abb.3).

Daraufhin erfolgte am 11.5.2010 die atypische Keilresektion von Unterlappen und Oberlappen rechts. Bei R0-Resektion wurde zu-

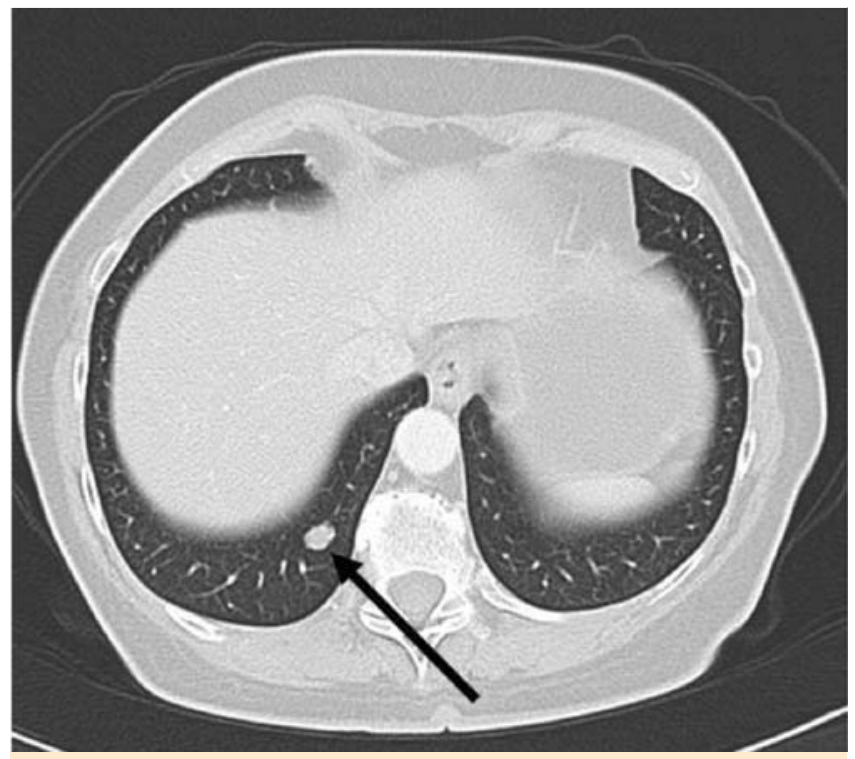

Abb.3 Nachfolgend Resektion von zwei pulmonalen Metastasen.

nächst, in der Tumorkonferenz vom 18.5.2010, das vierteljährliche Staging mittels PET-CT, MRT-Schädel, Lymphknotensonografie und laborchemischer Kontrolle (S100, LDH, alkalische Phosphatase) entschieden. Im Staging vom 2.8.2010 zeigten sich 2 neue pulmonale Metastasen, 12 Metastasen in beiden Leberlappen bis zu einer Größe von ca. $3 \mathrm{~cm}(\bullet$ Abb.4) sowie ein Herd mit tumortypisch gesteigerter Glukoseutilisation im Oberbauch rechts (Verdacht auf duodenale Metastasierung). Der S100-Wert stieg auf $0,15 \mu \mathrm{g} / \mathrm{l}$.

Auf eine Kombinationschemotherapie mit Carboplatin/Paclitaxel wurde verzichtet und wegen des hepatischen Stoffwechselweges wurde die Monochemotherapie mit Fotemustin $100 \mathrm{mg} / \mathrm{m}^{2}$ der Körperoberfläche (KO) favorisiert. 3 Monate nach Einleitung der Monochemotherapie mit Fotemustin zeigte sich eine partielle Remission mit Rückgang der Lebermetastasen. Bei der Patientin wurde eine Chemotherapie-induzierte Panzytopenie diagnostiziert, die mit Filgrastim, Darbepoetin alfa und teilweise mit Hämotherapie behandelt wurde. Im Zeitraum von 10/2010 bis 6/ 2011 erfolgten insgesamt 13 Gaben Fotemustin. Darunter zeigte sich zunächst eine partielle und nachfolgend (nach PET-CT-Kriterien) eine komplette Remission der Filialisierung ( $\bullet$ Abb.5, - Abb.6).

In der Tumorkonferenz 6/2011 wurde das Absetzen der Monochemotherapie bei kompletter Remission und das engmaschige Staging empfohlen.

Jedoch zeigte sich nach 10 Gaben Fotemustin ein plötzlich einsetzender und rasch progredienter, körperlicher und kognitiver Verfall mit demenziellen Symptomen wie Gedächtnisstörungen und Tremor.

Im August 2011 nach Beendigung der Chemotherapie wurde die Patientin zur Abklärung der neurologischen Symptomatik stationär aufgenommen. Nach Ausschluss anderer Ursachen wurde der Verdacht auf eine Fotemustin-induzierte Neurotoxizität gestellt. Daraufhin und bei Fortschreiten des kognitiven Verfalls erfolgte die Vorstellung der Patientin in einem spezialisierten Epilepsiezentrum. Die antikonvulsive Therapie wurde von Valproat (Orfiril $^{\circledR}$ ) auf Levetiracetam (Keppra ${ }^{\circledR}$ ) umgesetzt. Innerhalb eines kurzen Zeitraums bildete sich die dementielle Symptomatik komplett zurück. Mehr als $1 \frac{1}{2}$ Jahre nach Beendigung der Therapie 


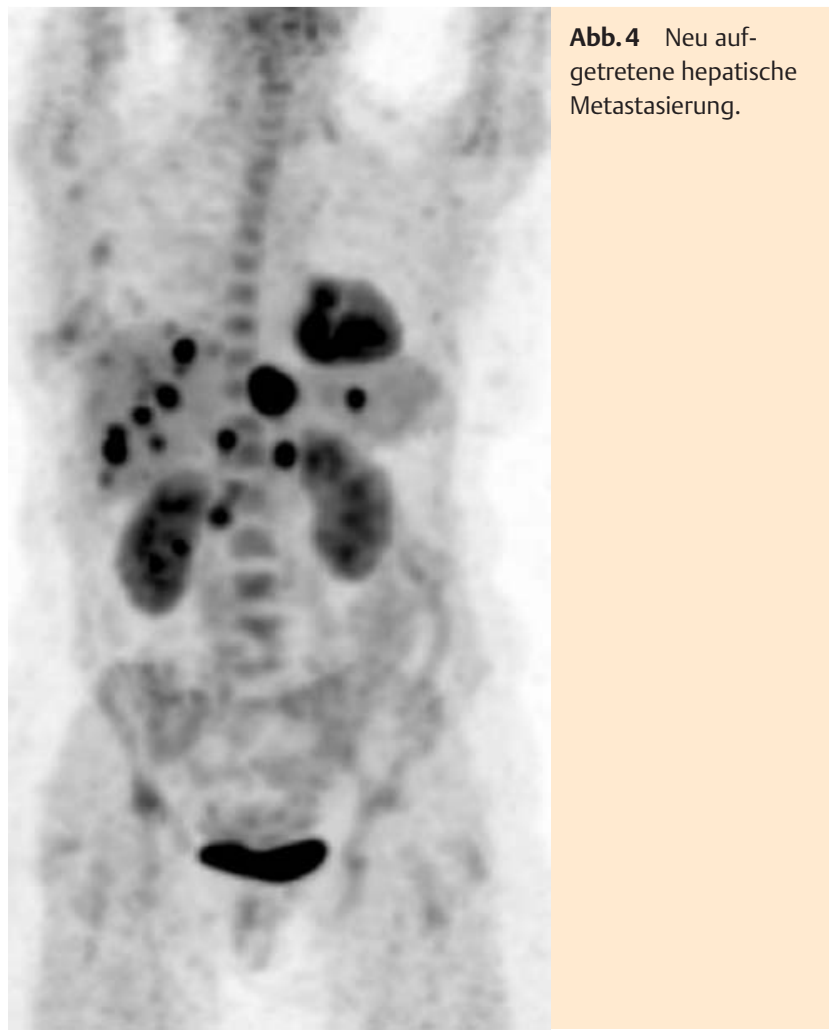

mit Fotemustin befand sich die Patientin noch in Vollremission und gutem Allgemeinzustand mit nebenwirkungsfreier Anti-Epilepsieeinstellung.

\section{Diskussion}

\section{$\nabla$}

Fotemustin ist eine Substanz aus der Gruppe der Nitrosoharnstoffe und war die erste Substanz mit einer nennenswerten Aktivität im zentralen Nervensystem. Es kann als First-line-Monochemotherapeutikum insbesondere bei zerebral metastasiertem Melanom eingesetzt werden. Jedoch treten unter Fotemustin häufig hämatologische Nebenwirkungen von Grad III und Grad IV auf [1]. Die neurologische Toxizität von Fotemustin ist eine sehr seltene Nebenwirkung. Vereinzelte Fälle wurden von Khalil

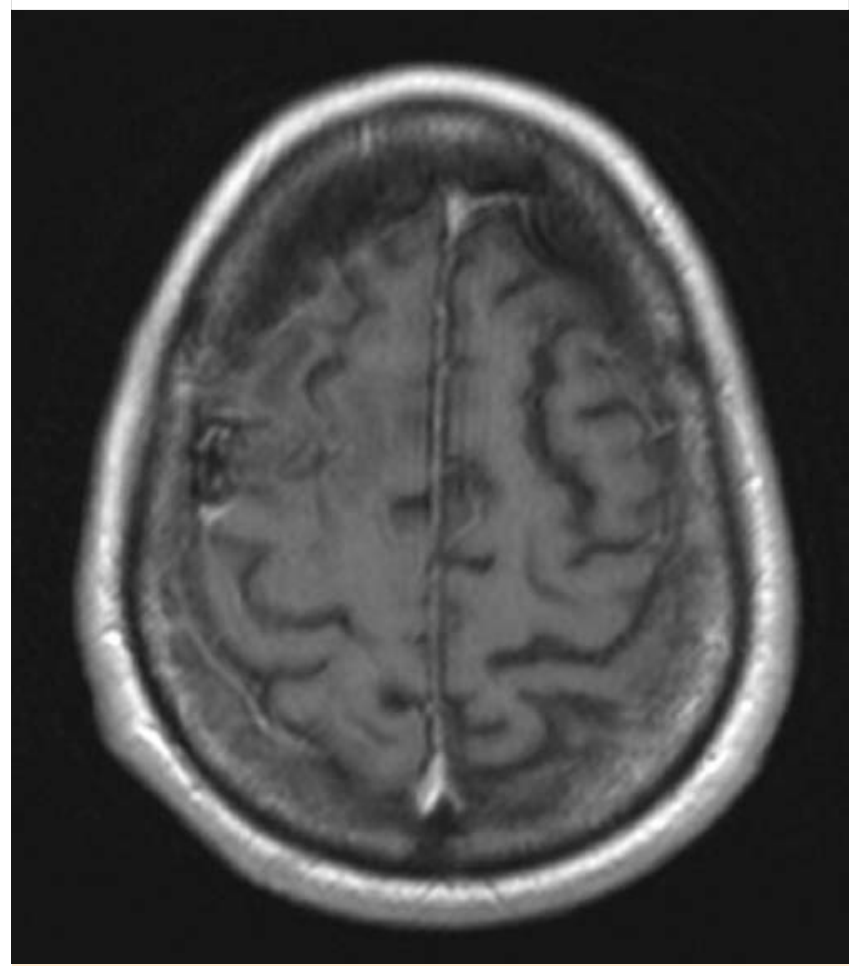

Abb. 6 Schädel-MRT von 8/2011: T1-Wichtung mit KM-Gabe.

et al. [2] und Gruss et al. [3] beschrieben. Gruss et al. berichteten über Hirnatrophie und Leukenzephalopathie bei einem männlichen Patienten mit zerebral metastasiertem Melanom, der über einen Zeitraum von 2 Jahren insgesamt 17 Gaben von Fotemustin kombiniert mit Ganzhirnbestrahlung erhalten hat [3]. Das Auftreten einer Fotemustin-assoziierten Enzephalopathie bei einer weiblichen Patientin mit nodulärem, malignem Melanom mit partieller Remission nach 10 Gaben von Fotemustin wurde von Khalil et al. beschrieben [2].

Valproat wird seit den 60er-Jahren im klinischen Alltag mit einem relativ zuverlässigen Nebenwirkungsprofil eingesetzt. Pankreatitis, Hepatotoxizität und Teratogenität sind die wichtigsten Nebenwirkungen dieser Substanz. Enzephalopathie in Assoziation mit Valproat ist zwar selten, stellt jedoch, wenn nicht
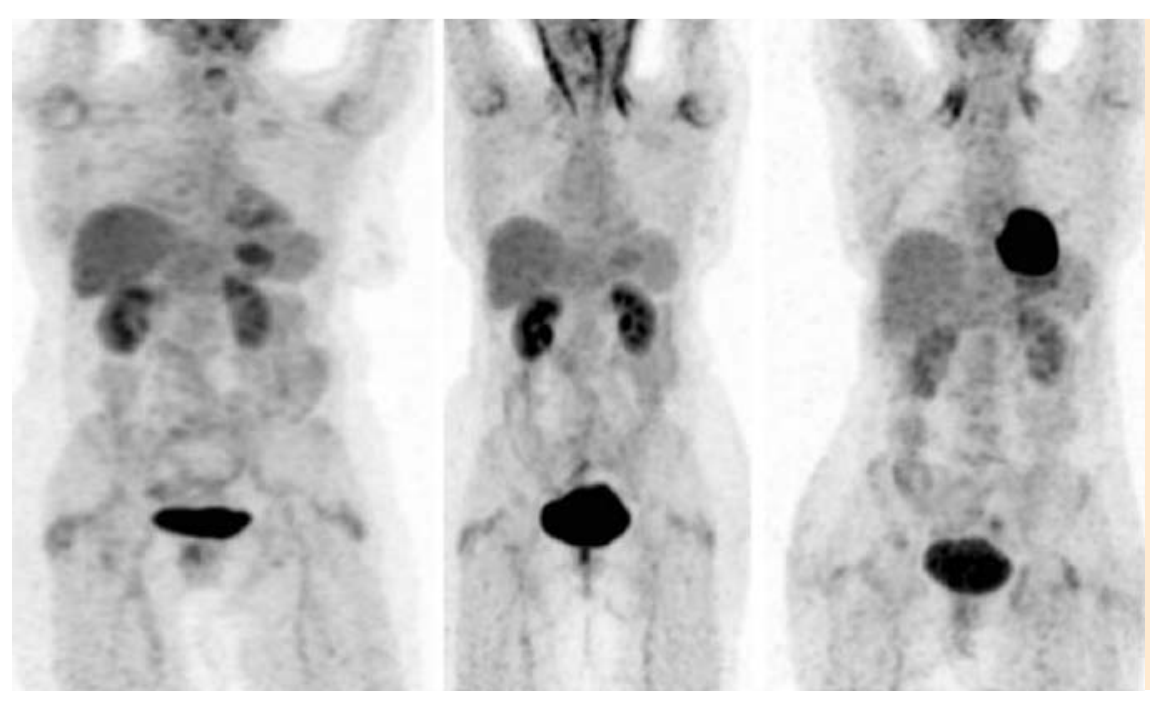

Abb.5 Keine Tumorprogression (von links nach rechts): PET-CT von 11/2010, 2/2011, 8/2011). 
zeitgerecht entdeckt, eine potenziell fatale Nebenwirkung von Valproat dar [4].

Gerstner et al. berichteten über 19 neue Fälle von 1994 bis 2003 in Deutschland mit Valproinsäure-induzierter Enzephalopathie [5]. Diese Fälle werden mit den 51 Fällen, die in der Literatur schon beschrieben wurden, zusammengerechnet. Parkinsonismus in Assoziation mit Valproat wurde auch beschrieben. Jedoch zeigte sich nach Erniedrigung der Dosierung eine Rückläufigkeit der Symptomatik [6].

In unserem Fall wurde die neurologische Symptomatik fälschlicherweise der Gabe von Fotemustin zugeordnet. Mit der Umsetzung von Valproat auf Levetiracetam trat jedoch eine komplette Rückläufigkeit der neurologischen Symptomatik auf $[8,9]$.

Die neurologische Toxizität ist eine sehr seltene Nebenwirkung von Fotemustin. Jedoch können auch die Begleitmedikationen die eigentliche Ursache eines schweren kognitiven Zerfalls darstellen. Die ausführliche Überprüfung der Nebenwirkungen der Co-Medikation gehört daher zu den primären Aufgaben des behandelnden Arztes [7]. Das frühzeitige Erkennen und die richtige Zuordnung der klinischen Symptomatik ermöglicht, wie in dem oben beschriebenen Fall, eine rechtzeitige und effektive therapeutische Intervention.

\section{Interessenkonflikt}

$\nabla$

Die Autoren geben an, dass kein Interessenkonflikt besteht.

Dieser Beitrag beinhaltet keine Studien an Menschen oder Tieren.

\section{Abstract}

\section{Long-Term Complete Remission of Cerebral, Pulmo- nary and Hepatic Metastases by Malignant Melanoma under Fotemustin with Reversible Dementia Syndrome Due to Valproic Acid}

A 63-year-old female patient suffers in 4/2010 for the first time a generalized epileptic seizure due to a single brain metastasis. In this patient a malignant melanoma was excised from the left elbow 40 years ago.

Apart from the brain metastasis we documented metastatic lesions in the lungs and the liver. The pulmonary and the brain metastases were resected. Furthermore the patient was prescribed valproic acid followed by the initiation of chemotherapy with fotemustine.

After the 10th application of fotemustine, the patient presented with sudden and rapidly progressive neurological disabilities. In 10/2011, after 13 applications of fotemustine, the patient was hospitalized in our neurological department. After the diagnostical assessment, the progression of the melanoma was excluded as a hypothesis for the neurological disabilities. An adequate reason for the dementia could not be found also. In reference to similar cases in the literature, a fotemustine-associated neurotoxicity was pointed out as the most probable diagnosis.

Despite that and because of the progression of the neurological symptoms, the patient attended a specialized epilepsy-centre. The anticonvulsant medication was changed. Instead of valproic acid, levetiracetam was applied. After the therapy adjustment the neurological symptoms disappeared.

More than $1 \frac{1}{2}$ year upon completion of the fotemustine chemotherapy, the patient remained in complete remission. This case report is an example for a successful therapy with fotemustine. The initially suspected side-effect of neurotoxicity was in this case correlated to another drug.

Literatur

1 Garbe C. Management des Melanoms. Heidelberg: Springer; 2006

2 Khalil Z, Pageot N, Carlander B et al. Neurological toxicity during metastatic melanoma treatment with fotemustine. Melanoma Res 2005; 15 : $563-564$

3 Gruss C, Geissler A, Schalke B et al. Severe neurological disabilities after complete remission of advanced malignant melanoma following fotemustine therapy in combination with total brain irradiation. Melanoma Res 2002; 12: $403-404$

4 Sousa $C$. Valproic acid-induced hyperammonemic encephalopathy - a potentially fatal adverse drug reaction. Springerplus 2013; 2: 13

5 Gerstner T, Buesing D, Longin E et al. Valproic acid induced encephalopathy - 19 new cases in Germany from 1994 to 2003 - a side effect associated to VPA-therapy not only in young children. Seizure 2006; 15: $443-448$

6 Chateauvieux S, Morceau F, Dicato $M$ et al. Therapeutic Potential and Toxicity of Valproic Acid. J Biomed Biotechnol 2010; 2010: 479364

7 Manckoundia P, Disson-Dautriche A, Rouaud O et al. Dementia syndrome in an elderly subject related to valproic acid use: a case report. Rev Med Interne 2008; 29: 827-829

8 Walstra GJ. Reversible dementia due to valproic acid therapy. Ned Tijdschr Geneeskd 1997; 141: 391 - 393

9 Vaquerizo J, Gómez Martín H, González Iglesias E et al. Reversible neuropsychological deterioration associated with valproat. Rev Neurol 1995; 23: $148-150$ 